\title{
Morphological variations of lung lobes and fissures: a preliminary study
}

\author{
Mathangasinghe Y, Samaranayake U M J E, Abarana K, Liyanage U A, Rajapakse Y N \\ Department of Anatomy, Faculty of Medicine, University of Colombo, Colombo, Sri Lanka
}

Keywords: Pulmonary; lobes; fissures; anatomy; morphology; cadaveric

\begin{abstract}
\section{Introduction}

Anatomical variations of the lung lobes and fissures have implications in diagnostic radiology and thoracic procedures. The prevalence of these variations is not reported in Sri Lanka.
\end{abstract}

\section{Methods}

A cross-sectional study was conducted on cadavers to assess the morphological variations of lung lobes and fissures. The presence and absence of lung lobes and fissures were reported in addition to describing the completeness of the major lung fissures.

\section{Results}

A total of 24 lungs in 12 cadavers were studied. Inferior accessory fissures were noted in one right and one left lung of two independent cadavers. Two cadavers had no horizontal fissures, and one left lung had an incomplete oblique fissure posteriorly. Azygos lobe was not observed in this study sample.

\section{Conclusions}

The morphology of lung lobes and fissures demonstrate a notable variability, on par with previous reports. Further studies with a large sample size in our population are necessary for generalizable results.

\section{Introduction}

During the embryonic development of the lung, the visceral pleura folds into the obliterating spaces surrounding lobar bronchi, forming lung fissures. The oblique fissure of the left lung separates it to the upper and lower lobes. The right oblique fissure separates the right middle lobe from the right lower lobe while the horizontal fissure separates the right upper lobe from the right middle lobe. However, the separation of these lobes by the said fissures is often

\section{Correspondence: Mathangasinghe Y}

E-mail: yasith@anat.cmb.ac.lk

(iD) https://orcid.org/0000-0003-4641-5642

Received: 21-08-2021 Accepted: 22-11-2021

DOI: http://doi.org/10.4038/sljs.v39i3.8884 incomplete in contrast to the textbook descriptions [1]. Moreover, accessory fissures are formed due to the anomalous development of the lung. The presence of incomplete or accessory fissures is associated with the anatomical variations of the lung lobes, which adds to the complexity of the lung morphology.

Knowledge of the anatomical variations of the lungs is important for the radiologists to interpret radiographs, for the thoracic surgeons to plan segmental resections/lobectomies and for the bronchoscopists in minimally invasive endoscopic procedures. The reported prevalence of morphological variations of the lung lobes and fissures vary widely in different populations [1]. Despite these variants being well reported in the literature, to our knowledge, there are no publications about a Sri Lankan population. Thus, in this preliminary study, we intended to describe the frequency of morphological variations of the lung lobes and fissures in a Sri Lankan population.

\section{Methods}

This descriptive cross-sectional study was conducted at the Department of Anatomy, Faculty of Medicine, University of Colombo. Consent to conduct studies on the self-donated cadavers was obtained from the next of kin. The study protocol conformed to the guidelines set out by the Declaration of Helsinki.

A sample of adult cadavers of Sri Lankan origin was selected using simple random sampling. Cadavers with a history of lung malignancies, pulmonary tuberculosis, congenital malformations, and those who underwent thoracic surgeries were excluded from the study. Cadavers were fixed with phenoxyethanol mix as described in detail elsewhere [2]. Two incisions were made bilaterally along the mid-axillary lines. The anterior thoracic cage was removed after cutting the clavicles at their midpoints and ribs along the mid-axillary lines. Bronchi were transected approximately $1 \mathrm{~cm}$ proximal to the hila and the lungs were removed from the thoracic cavities. Subsequently, lungs were inspected for anatomical variations. Fissures were categorized as complete, incomplete, or absent. The presence or absence of accessory fissures or lobes were also reported. 


\section{Results}

A total of 24 lungs in 12 cadavers were studied. Male: female ratio was 1:1. The age of the cadavers ranged from 34 to 86 years. The anatomical variations of major lung fissures and lobes in the study population and a gender-based contingency table are provided in Table 1 and Supplementary Table 1, respectively. Inferior accessory fissures were noted in one right and one left lung of two different cadavers [Figure 1]. Two cadavers had no horizontal fissures [Figure 2], and one left lung had an incomplete oblique fissure posteriorly [Figure 3]. Azygos lobe was not observed in this study sample.

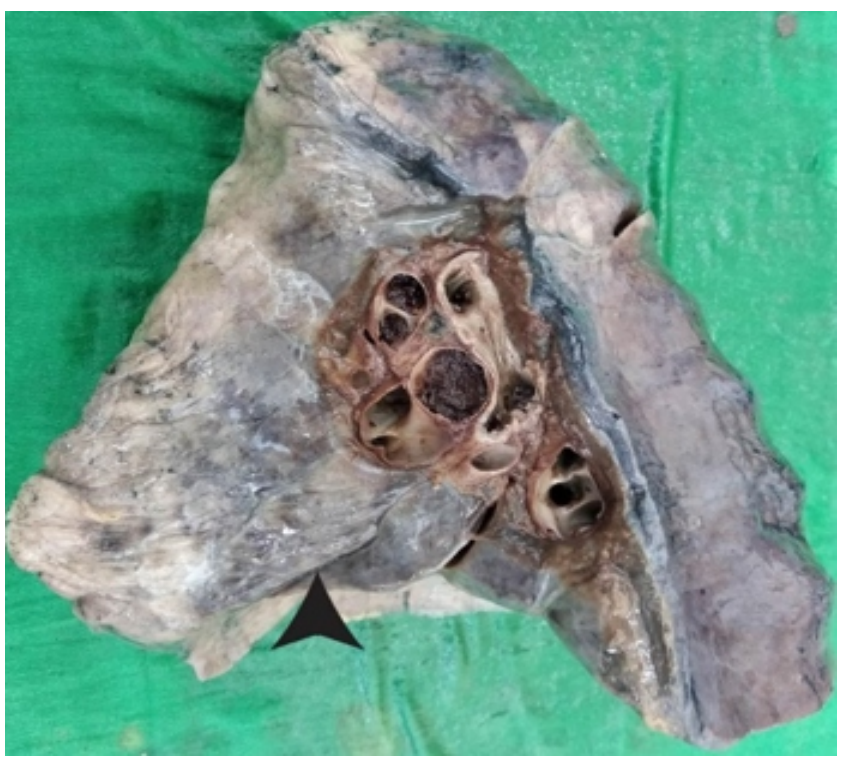

Figure 1. Inferior accessory fissure [black arrowhead].

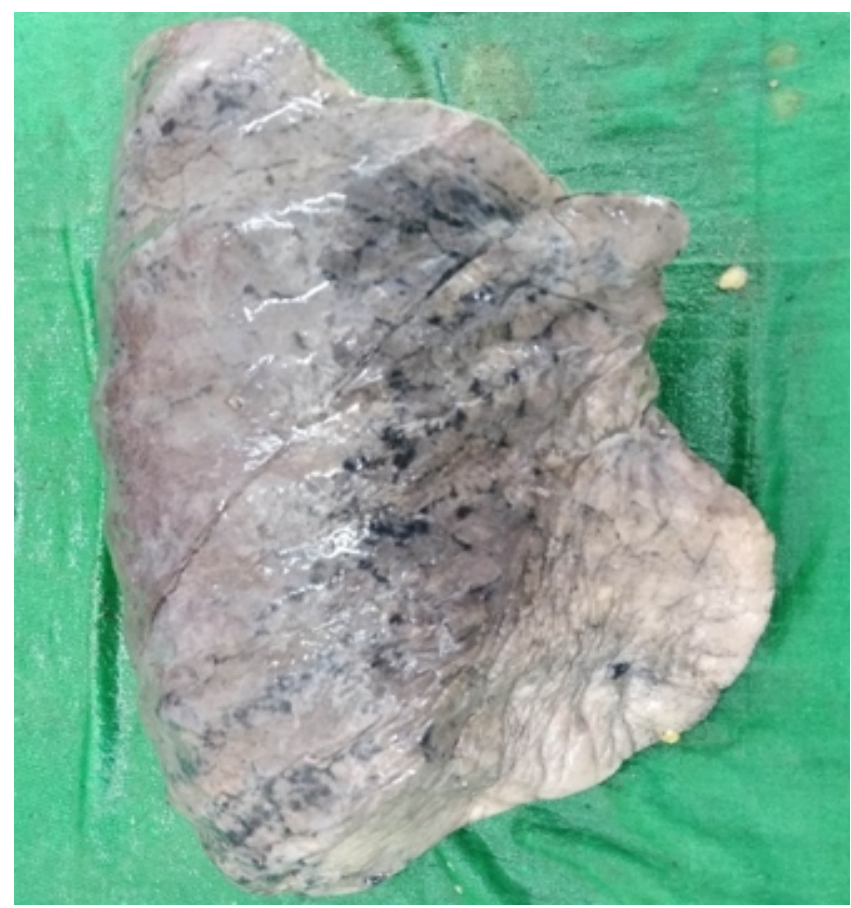

Figure 2. Absent horizontal fissure

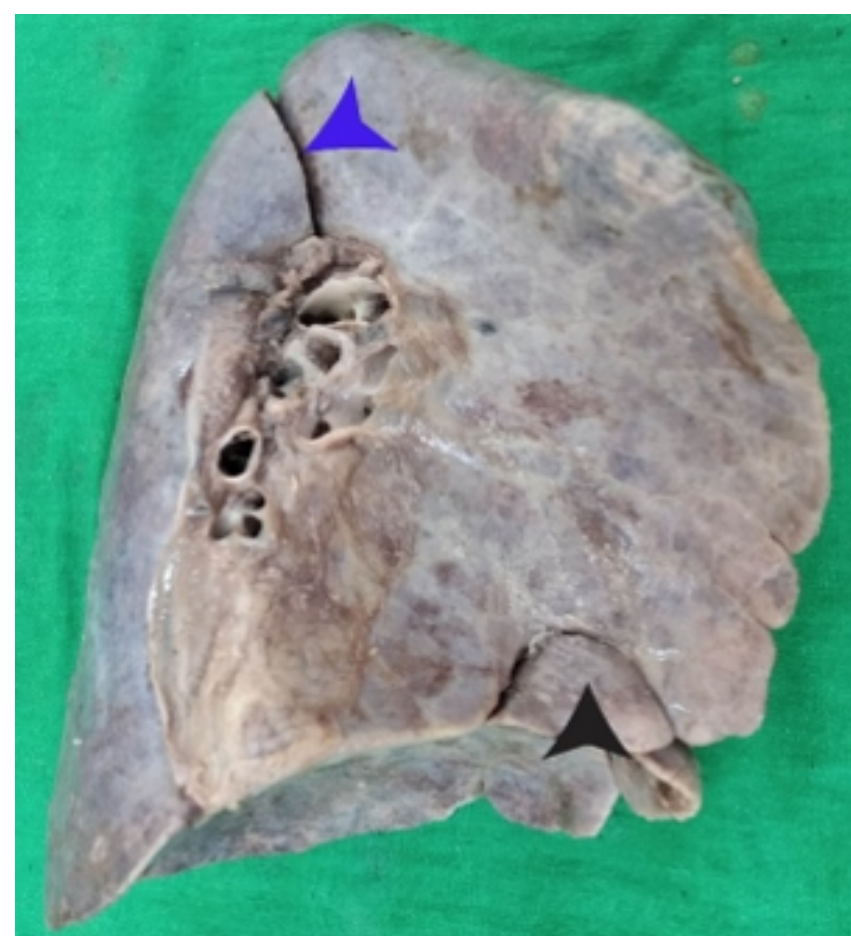

Figure 3. Incomplete oblique fissure [blue arrowhead] and an accessory lobe [black arrowhead]

Table 1.Variations of the major lung fissures and lobes $[\mathrm{N}=12$ patients $]$

\begin{tabular}{|l|l|l|l|l|}
\hline & & & Right Lung & Left Lung \\
\hline Fissures & Horizontal & Incomplete & 0 & N/A \\
\hline & & Absent & $2[17 \%]$ & N/A \\
\hline & Oblique & Incomplete & 0 & $1[8 \%]$ \\
\hline & & Absent & 0 & 0 \\
\hline & Accessory Fissures & $1[8 \%]$ & $1[8 \%]$ \\
\hline Lobes & \multicolumn{2}{|l}{ Accessory lobes } & $3[25 \%]$ & $1[8 \%]$ \\
\hline
\end{tabular}

\section{Discussion}

Pioneering cadaveric studies by Medlar in 1947 shed light on morphological variations of the lung lobes and fissures [3]. With the understanding of the importance of such variations in planning thoracoscopic surgeries, numerous anatomists and clinicians attempted to consolidate the patterns of these morphological variations. To provide a framework for such surgical procedures, in 1997, Craig and Walker proposed an extensive anatomical classification of the pulmonary fissures [4]. This comprehensive classification incorporated not only the presence or absence of fissures but also their completeness.

In our study, oblique fissures were present in all cadavers, with only one left oblique fissure being incomplete. This finding is consistent with the majority of previous reports, where oblique fissure was consistently noted, with at least $50 \%$ of the oblique fissures being complete [see Supplementary Table 2]. However, incompleteness of the 
oblique fissure was more commonly noted anteriorly $[1,5]$ in contrast to our finding where it was deficient posteriorly. The frequency of absent horizontal fissure was on par with the previous reports.

The commonest morphological variation of the lung is accessory fissures [6]. We noted $8 \%$ of the cadavers had accessory fissures, while all of them were inferior accessory fissures surrounding the medial basal segment [S7] of the lung. This, too, is in accordance with the literature where inferior accessory fissures are found commoner than superior accessory fissures [6-8]. Intriguingly, however, we did not observe left accessory fissures delineating azygous lobes in our study despite it being reported to be very common in certain populations $[1,8,9]$.

Knowledge of common anatomical variations of the lung lobes and fissures is important for radiologists since the presence of accessory and incomplete fissures are well known for confusions in interpreting radiological images, particularly pleural effusions and mass lesions $[6,10,11]$. The appearance of fluid tracking may be obscured along the incomplete fissures in pleural effusion [12]. Diagnosis of endobronchial mass lesions may be delayed in the presence of incomplete fissures where the distal lung collapse may not occur when the adjacent lung segments are communicating with each other through the incomplete fissure [11], a phenomenon known as "collateral air drift" [10]. Accessory lung fissures could be mistaken for consolidation or atelectasis.

Anatomical variations of the fissures and lobes have implications in segmental resections and lobectomies. The degree of completeness of lung fissures is reported to be associated with short-term postoperative complications following segmental resections and lobectomies for nonsmall cell carcinoma of the lung [13] and minimally invasive endoscopic procedures for emphysema [14]. Notably, incomplete major lung fissures lead to air leaks following segmental resection [10]. Thus, the presence of incomplete lung fissures warrants the thoracic surgeon to examine these regions carefully during lung resections to ensure the incomplete fissures are stapled adequately when separating conjoint lobes [11]. Similarly, additional lobectomies may be necessary during lung resections for malignant neoplasms if the tumour occurs in a region with an incomplete fissure [1]. The presence of incomplete fissures is also associated with poor long-term surgical outcomes of curative resections for early adenocarcinoma of the bronchus [9, 15]. Moreover, infections could easily spread between the adjacent lobes due to the presence of incomplete fissures [11].

\section{Limitations}

There is an ongoing debate where post-mortem changes and fixation with formaldehyde could lead to overestimation of the incompleteness of the lung fissures [1]. The results of phenoxyethanol fixatives on this are less clear. Because of this reason, we did not intend to report the absolute lengths or the degree of incompleteness of the fissures. Moreover, this was a preliminary study, where the sample size was limited.

\section{Conclusions}

The morphology of lung lobes and fissures demonstrate a notable variability, in par with previous reports. Further studies with a large sample size in our population are necessary for generalizable results.

All authors disclose no conflict of interest. The study was conducted in accordance with the ethical standards of the relevant institutional or national ethics committee and the Helsinki Declaration of 1975, as revised in 2000.

\section{References}

1 West C T, Slim N, Steele D, Chowdhury A, Brassett C. Are textbook lungs really normal? A cadaveric study on the anatomical and clinical importance of variations in the major lung fissures, and the incomplete right horizontal fissure. Clinical Anatomy. 2021;34[3]:387-396.

https://doi.org/10.1002/ca.23661

2. Anthony DJ, Mathangasinghe Y, Samaranayake UM, Basnayake BM, Malalasekera AP. Pigmented border as a new surface landmark for digital nerve blocks: a cross sectional anatomical study. Journal of Hand Surgery [European Volume]. 2019;44[9]:932-936.

https://doi.org/10.1177/1753193419845291

3. Medlar E. Variations in interlobar fissures. Am J Radiol. 1947;57:723-725.

4. Craig S, Walker W. A proposed anatomical classification of the pulmonary fissures. Journal of the Royal College of Surgeons of Edinburgh. 1997;42[4]:233-234.

5. Dutta S, Mandal L, Mandal SK, Biswas J, Ray A, Bandopadhyay M. Natural fissures of lung: anatomical basis of surgical techniques and imaging. Natl J Med Res. 2013;3:117-121.

6. Yıldız A, Gölpınar F, Çalıkoğlu M, Duce MN, Özer C, Apaydın FD. HRCT evaluation of the accessory fissures of the lung. European journal of radiology. 2004;49[3]:245-249. https://doi.org/10.1016/S0720-048X(03)00137-2

7. Godwin JD, Tarver RD. Accessory fissures of the lung. American journal of roentgenology. 1985;144[1]:39-47. https://doi.org/10.2214/ajr.144.1.39

8. Nene AR, Gajendra KS, Sarma MVR. Lung lobes and fissures: a morphological study. Anatomy: International Journal of Experimental \& Clinical Anatomy. 2011;5. https://doi.org/10.2399/ana.10.005

9. Akhtar J, Lal A, Martin KB, Popkin J. Azygos lobe: A rare cause of right paratracheal opacity. Respiratory medicine case reports. 2018;23:136-137. https://doi.org/10.1016/j.rmcr.2018.02.001 
10.Hayashi K, Aziz A, Ashizawa K, Hayashi H, Nagaoki K, Otsuji H. Radiographic and $\mathrm{CT}$ appearances of the major fissures. Radiographics. 2001;21[4]:861-874.

https://doi.org/10.1148/radiographics.21.4.g01j124861

11. Tarver RD. How common are incomplete pulmonary fissures, and what is their clinical significance? AJR American journal of roentgenology. 1995;164[3]:761-761.

https://doi.org/10.2214/ajr.164.3.7863909

12. Raasch B, Carsky E, Lane E, O'Callaghan J, Heitzman E. Pleural effusion: explanation of some typical appearances. American Journal of Roentgenology. 1982;139[5]:899-904.

https://doi.org/10.2214/ajr.139.5.899

13.Li S, Wang Z, Zhou K, et al. Effects of degree of pulmonary fissure completeness on major in-hospital outcomes after videoassisted thoracoscopic lung cancer lobectomy: a retrospectivecohort study. Therapeutics and clinical risk management. 2018;14:461. https://doi.org/10.2147/TCRM.S159632
14.Koster TD, Slebos D-J. The fissure: interlobar collateral ventilation and implications for endoscopic therapy in emphysema. International journal of chronic obstructive pulmonary disease. 2016;11:765. https://doi.org/10.2147/COPD.S103807

15.Lee S, Lee JG, Lee CY, Kim DJ, Chung KY. Pulmonary fissure development is a prognostic factor for patients with resected stage I lung adenocarcinoma. Journal of surgical oncology. 2016;114[7]:848-852.

https://doi.org/10.1002/jso.24438 